\title{
COVID-19: Pandemie, Infodemie?
}

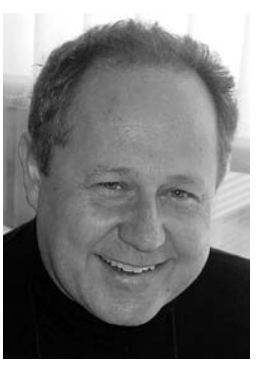

Manfred Wildner
Bibliografie

Gesundheitswesen 2021; 83: 247-249

DOI 10.1055/a-1391-3121

ISSN 0941-3790

(C) 2021. Thieme. All rights reserved.

Georg Thieme Verlag KG, Rüdigerstraße 14,

70469 Stuttgart, Germany

Korrespondenzadresse

Prof. Dr. med. Manfred Wildner

Pettenkofer School of Public Health

c/o Bayerisches Landesamt für Gesundheit und Lebensmittelsicherheit

85764 Veterinärstraße 2

Oberschleißheim

Deutschland

Manfred.Wildner@Igl.bayern.de
„Eine Infodemie ist ein Übermaß an angebotener Information, sowohl offline [in den klassischen Medien] als auch online“, stellen die internationalen Organisationen WHO, UN, UNICEF, UNDP, UNESCO, UNAIDS, ITU, UN Global Pulse und IFRC in einer seltenen gemeinsamen Erklärung fest [1]. Sie kommen dann schnell auf den Punkt: Darin enthalten seien "gezielte Versuche, Falschinformationen zu verbreiten, um die öffentlichen Abwehr- und Hilfsmaßnahmen zu unterminieren und alternative Agenden zu befördern“. Solche Fehlinformationen könnten dann gesundheitliche Folgen haben, zu Stigmatisierungen und zur Ablehnung von sinnvollen Infektionsschutzmaßnahmen führen, einschließlich von Hygienemaßnahmen, Test- und Impfangeboten. Kurz: Falschinformationen können Menschenleben kosten, insbesondere in einer Pandemie und darüber hinaus durch Hetze und Misstrauen den sozialen Zusammenhalt einer Gemeinschaft nachhaltig schädigen. Damit wird die Pandemie in einem zweiten Schritt zum Vehikel einer nochmals die Gesundheit auf individueller und Bevölkerungsebene schädigenden „Infodemie“.

Auf die Charakterisierung einer Epidemie bzw. Pandemie als ganz wesentlich auch sozial(systemisch)es Phänomen war schon hingewiesen worden [2]. Ein Paradoxon angesichts der doch vordergründig klaren biomedizinischen Zusammenhänge mit einem biologischen Mikroorganismus und dessen Auswirkungen auf einen ebenfalls biologisch verstehbaren Wirtsorganismus? Auch wenn diese systemische Charakterisierung zunächst nur in dem engeren Zusammenhang der epidemiologischen Triade aus Erreger (Agent), Wirt (Host) und natürlicher, technischer und sozialer Umwelt (Environment) zu verstehen ist, bleibt sie auch in einem weiteren Rahmen für die diversen sozialen Systeme gültig. Ein Grund, sich diesen weiteren Rahmen näher anzusehen.

Um bei der Bezeichnung „Infodemie“ zu beginnen - hier sind die Wortbestandteile der „Information“ und der „demie“, also dessen, was sich im Volk (demos) gleichsam krankmachend verbreitet, zu betrachten. Bezogen auf die Information lässt sich ein Miss- brauch von Information, also ein fehlgeleiteter Zweck oder auch eine gezielte Falschinformation, von einem „Zuviel“ an Information, einem information overload abgrenzen. Hinzu kommen noch individuelle Reaktionen des Informationsempfängers auf die dargebotene Information im Sinne einer thematischen Ermüdung (information fatigue) oder auch einer Abwehrreaktion auf Grund fehlender emotionaler Ansprache.

Dass die oft nur schwer sichtbaren Krankheitsursachen sowohl zu ihrer Leugnung (Denialism) wie auch zur Erfindung phantasievoller Ätiologien und Pathogenesen nebst zugehöriger, finanziell einträglicher Diagnose- und Therapieangebote führen können, ist eine buchstäblich leidvolle, hinlänglich bekannte Tatsache [3, 4]. Die Informationskrise einer Infodemie beschränkt sich allerdings nicht nur auf eine fehlende Gesundheitsbildung und Gesundheitskompetenz allein, als Hintergrund für windige und potenziell schädigende Akteure wie in alten Zeiten: „Ich bin der Doktor Eisenbart, Widewidewitt, bum, bum, kurier` die Leut auf meine Art, Widewidewitt, bum, bum... “ Unseriöse Krankheitserfindungen und Ursachenzuschreibungen können in der Gegenwart auch angestrebtes Ergebnis strategisch-planvollen Handelns von grundsätzlich sehr gut informierten Interessensgruppen sein [5-7].

Bisher waren solche Fehlinformationen zumeist Ereignisse im Binnenraum des Gesundheitswesens, Aktivitäten von Personen und Institutionen mit wirtschaftlichen Interessen innerhalb des Systems. Auch dies findet sich in der Coronapandemie 2020/21 wieder: Neben der Leugnung der Pandemie das Anpreisen von diagnostischen Wunderwaffen mit weit überzogenen Behauptungen zu deren Leistungsfähigkeit, die Verbreitung unseriöser therapeutischer Heilsversprechen im Bereich der Allopathika, Homöopathika und rein pflanzlichen Produkte oder auch das smarte Besetzen von Nischen und das Ausnutzen von Gelegenheiten mit den unerwünschten Effekten von gleichzeitiger Über-, Unter- und Fehlversorgung auf Systemebene. Und auch die seriösen Handlungsop- 
tionen, z. B. Impfangebote oder Schutzmasken, können durchaus unseriös gehandelt werden: mit rhetorisch und rechtlich verklausulierten, kalkulierten Vertragsbrüchen, nur schwach verhohlenen Eigeninteressen bei der Preisfindung und unlauteren Gewinnerwartungen bei den Mittelsmännern und -frauen.

Was uns die Infodemie darüber hinaus nun neu beschert, sind weitreichendere gesellschaftspolitische Einflussnahmeversuche. Um hier nur beispielhaft die Bewegung der „Querdenker“ herauszugreifen: „Es handelt sich nicht um eine, sondern um mehrere, häufig disparate soziale Gruppen, die über geteilte Mentalitäten verbunden sind“, so die Basler Soziologiestudie von Oliver Nachtwey. „Charakteristisch für diese neue Bewegung ist eine starke Entfremdung von den Institutionen des politischen Systems, den etablierten Medien und - zumindest für Deutschland - den alten Volksparteien“ [8]. Und so werden denn auch die Methoden der Anthropologie und Ethnologie bemüht, um Erkenntnisse über diesen inmitten einer hypermodernen Gesellschaft zunächst so fremden, offenbar neu entstandenen „Stamm“ zu gewinnen und Ansätze zu dessen Verständnis zu bekommen. Ein Teil des Fazits: „Die Entfremdung von der industriell geprägten und durchrationalisierten Hypermoderne zeigt sich nicht nur in der Skepsis gegenüber ihren Institutionen, wie z. B. den Parteien, sondern auch bezüglich einer romantisch inspirierten Hinwendung zu ganzheitlichen, anthroposophischen Denkweisen, dem Glauben an die natürlichen Selbstheilungskräfte des Körpers, Forderungen nach mehr spirituellem Denken und dem Wunsch, Schulmedizin und alternative Heilmethoden gleichzustellen“ [8, ebd.]. Ob diese beklagte Skepsis immer so ganz grundlos ist, sei angesichts des durchaus auch diskussionswürdigen Agierens von etablierten Personen und Parteien im gesellschaftlichen Mainstream dahingestellt.

Sind viele Menschen der Moderne von eben dieser Moderne vielleicht überfordert und ziehen sich in eine Vormoderne zurück, kaschiert als Postmoderne? Der Mensch sei ein Augentier, aber das Virus lasse sich so einfach nicht sehen, räsoniert der Philosoph Albert Newen dazu. Wissenschaft werde von Populisten zunehmend in Verruf gebracht, welche „das Volk“ gegen „die Elite“ in Stellung bringen, meinen andere [9]. Dritte räsonieren über die Rolle des Wissenschaftsjournalismus und diagnostizieren einen tiefgehenden Vertrauensverlust zwischen Wissenschaft und Politik [10]: Der Weg von den Daten zur Information, vom Wissen bis zum Bewusstsein scheint länger - oder vielleicht auch verstopfter - geworden zu sein.

Hier sind wir auch schon bei dem Wortbestandteil der krankmachenden Info-, demie“ angelangt: Jede Krise ist (auch) eine Informationskrise [11]. Kann uns denn die Wissenschaft aus dieser Krise retten - oder kämpft die Wissenschaft womöglich derzeit mit sich selbst? Sind wissenschaftliche Publikationen als Basis des Wissen-Schaffens dabei, durch ihre vorgezogene Verfügbarkeit in Preprint-Archiven und ihre Referenzierung schon als Vorab-Publikationen in ihrem Kern, nämlich der kritischen Überprüfung ihrer Methoden und Ergebnisse im kollegialen Fachkreis vor einer Publikation, Schaden zu nehmen? Neben eine der Wissenschaft inhärenten epistemische Unsicherheit treten im 21. Jahrhundert noch die enorme Geschwindigkeit des Informationszuwachses, die teilweise divergierenden politischen Bewertungen von wissenschaftlichen Erkenntnissen sowie die weitgehende Regelfreiheit der neuen sozialen Medien und Informationskanäle [12]. Letztere ermöglichen wissenschaftliche Halbwelten und ihre Protagonisten und Heroen, verkannten Lichtgestalten und tragischen Helden, welche in ihrer Herkunftsdisziplin von der Petrischale bis zur Alternativmedizin reichen, vom Professor emeritus bis zu den Millionen an virologisch-epidemiologischen (Neu-)Experten in der allgemeinen Wohnbevölkerung. In der Folge reflektieren denn auch die Wissenschaftsphilosophen, in wieweit sie ihre fachspezifischen Wachund Achtsamkeitspflichten verletzt haben könnten - zunächst als preprint, versteht sich [13]. Ein gewisser Trost ist da die Feststellung: „Der wissenschaftliche Diskurs kennt nicht nur die Zustände „wahr“ und „falsch“, sondern führt in vielen Fällen eher mäandrisch zu einem Zugewinn an Wissen“ [14]. Diese richtige Beobachtung sollte jedoch den notwendigen einfachen, klaren und praxistauglichen Botschaften im Rahmen der Pandemiebewältigung nicht entgegenstehen [15].

In diesem Sinne möchten auch die Berichte in diesem Heft wieder ihren Beitrag leisten: Zu primärärztlichen Strategien und Zusammenarbeit während der ersten Phase der COVID-19-Pandemie in Baden-Württemberg, zum Management der ersten COVID-19 Welle in Kinder- und Jugendarztpraxen im Saarland, zur Entwicklung der Notaufnahmefallzahlen eines Schwerpunktversorgers im Verlauf der Corona-Pandemie in Mecklenburg-Vorpommern, zur Angst vor dem Coronavirus und der Absicht zum Befolgen der AHARegeln und Risikowahrnehmung bezüglich Arztbesuchen bei psychisch vorerkrankten Menschen, zu Auswirkungen der COVID-19 Pandemie auf die medizinische Versorgung von Patienten mit angeborenen Blutungsneigungen, zur Herzinfarktsterblichkeit innerhalb und außerhalb der Kliniken in Berlin, zur Integrierten Stadtentwicklung und Gesundheit am Beispiel Hamburgs und zur subjektiven Hitzebelastung als Folge des Klimawandels in konträren Stadtquartieren unter Aspekten der Umweltgerechtigkeit. Hinzu kommt ein Positionspapier des Deutschen Netzwerks Versorgungsforschung (DNVF) zur anwendungsbegleitenden Datenerhebung nach Sozialgesetzbuch V.

Um am Ende noch einmal auf den Anfang zurückzukommen: Auch die Erforschung der Infodemie als solcher erfordert wissenschaftliche Anstrengungen. Empfehlungen dazu sind gemacht [16]: Die Messung und das Monitoring des Impacts einer Infodemie während einer gesundheitlichen Notlage, ihre Aufdeckung und Nachverfolgung, die Formulierung von Gegenmaßnahmen als Antwort mit geeigneten schützenden und den Schaden einer Infodemie eingrenzenden Interventionen, deren Evaluation und die darauf aufsetzende weitere Stärkung der Resilienz von Individuen und Gemeinschaften gegen Infodemien, einschließlich der gezielten Förderung der Entwicklung, Adaptation und Anwendung neuer Werkzeuge. Und so wird am Ende dann wohl (gute) Information gegen (schlechte) Information stehen, (vernünftige) Meinung gegen (unvernünftige) Meinung, (belastbare) Evidenz gegen Evidenz (mit Bias) - diese Auseinandersetzung wird das bessere (Ge-) Wissen hoffentlich gewinnen und könnte damit dann auch eine neue gesellschaftliche Immunität zurücklassen. Diese infodemische Immunität würde so die pandemische Immunität gegenüber dem biologischen Krankheitserreger ergänzen - „Auffrischimpfungen“ im Intervall sollten in beiden Fällen vorgesehen werden. 


\section{Interessenkonflikt}

Die Autorinnen/Autoren geben an, dass kein Interessenkonflikt besteht.

\section{Literatur}

[1] Managing the COVID-19 infodemic: Promoting healthy behaviours and mitigating the harm from misinformation and disinformation (who. int), accessed 04.03.2021

[2] Wildner M. Epidemien und gesellschaftliches Leben. Gesundheitswesen $2021 ; 83: 5-7$

[3] Wildner M. Kennen Sie Morgellonen? Gesundheitswesen 2009; 71: 795-796

[4] Diethelm P, McKee M. Denialism: what it is and how should scientists respond? Eur J Public Health 2009; 19: 2-4

[5] Paccaud F. Implausible diseases and public health. Eur ] Public Health 2007; 7: 410

[6] Payer L. Disease mongers. New York: John Wiley; 1992

[7] Moynihan R, Heath I, Henry D et al Selling sickness: the pharmaceutical industry and disease mongering. Commentary: medicalisation of risk factors. Br Med J 2002; 324: 886-891

[8] Nachtwey O, Schäfer R, Frei N. Politische Soziologie Der Corona-proteste. SocArXiv. December 20 2020; URL: doi:10.31235/osf.io/zyp3f
[9] Mede NG, Schäfer MS. Science-related populism: Conceptualizing populist demands toward science. Public Understanding Science 2020; 29: $473-491$

[10] Stollorz V. Herausforderungen für den Journalismus über Wissenschaft in der Coronapandemie - erste Beobachtungen zu einem Weltereignis. Bundesgesundheitbl 2021; 64: 70-76

[11] Christof C, Nußbaumer-Streit B, Gartlehner G. Risikokommunikation in gesundheitsbedrohlichen Krisensituationen: Zusammenfassung einer WHO-Leitlinie. Gesundheitswesen 2019; 81: 846-849

[12] Horton R. Offline: Science and politics in the era of COVID-19. Lancet 2020; 396: 1319

[13] van Basshuysen P, White L. (2021) How Philosophers of Science Violated Their Epistemic Duties During the SARS-CoV-2 Crisis. [Preprint]. URL: http://philsci-archive.pitt.edu/id/eprint/18584 accessed 2021-03-04

[14] Fiack S, Koch-Gromus U, Kuhn J et al. Medizin und Medien - Symbiose oder Konflikt? Bundesgesundheitsbl 2021; 64: 1-2. https://doi. org/10.1007/s00103-020-03262-0

[15] Rosen B, Waitzberg R, Israeli A. Israel's rapid rollout of vaccinations for COVID-19. Israel J Health Pol Res 2021; $10: 6$

[16] WHO public health research agenda for managing infodemics. Geneva: World Health Organization; 2021 